\title{
Local Logics, Non-monotonicity and Defeasible Argumentation
}

\section{GUSTAVO A. BODANZA}

Departamento de Humanidades, Universidad Nacional del Sur, 8000 Bahía Blanca, Argentina

E-mail: ccbodanz@criba.edu.ar

FERNANDO A. TOHMÉ

Departamento de Economía, Universidad Nacional del Sur, 8000 Bahía Blanca, Argentina

CONICET

E-mail: ftohme@criba.edu.ar

November 2004

Abstract. In this paper we present an embedding of abstract argumentation systems into the framework of Barwise and Seligman's logic of information flow. We show that, taking P.M. Dung's characterization of argument systems, a local logic over states of a deliberation may be constructed. In this structure, the key feature of non-monotonicity of commonsense reasoning obtains as the transition from one local logic to another, due to a change in certain background conditions. Each of Dung's extensions of argument systems leads to a corresponding ordering of background conditions. The relations among extensions becomes a relation among partial orderings of background conditions. This introduces a conceptual innovation in Barwise and Seligman's representation of commonsense reasoning.

Keywords: local logics, non-monotonicity, defeasible argumentation.

(c) 2006 Kluwer Academic Publishers. Printed in the Netherlands. 
Keywords: local logics, non-monotonicity, defeasible argumentation.

\section{Introduction}

The modeling of commonsense reasoning has been a goal of Artificial Intelligence since its inception almost fifty years ago (McCarthy and Hayes, 1969). This project has been hampered by logical limitations, one of the most important being that, in contrast to first order logic, commonsense reasoning is non-monotonic. That is, it allows for changes in the conclusions of a line of reasoning as a result of the incorporation of new information.

Many alternative forms of representing non-monotonicity have been advanced since the end of the 1970s, when researchers in AI realized that classical formal logic was not enough to represent the reasoning abilities of intelligent agents (MacDermmot and Doyle, 1980),(McCarthy, 1980),(Reiter, 1980). One approach which was particularly successful in incorporating non-monotonicity while at the same time retaining other features of commonsense reasoning was argumentation systems (Loui, 1987), (Pollock, 1987), (Poole, 1988), (Lin and Yoham, 1989), (Simari and Loui, 1992). These systems capture quite accurately the dynamics of deliberation, in which agents weigh the pros and cons of the conclusions of different lines of reasoning, and end up choosing the "strongest". The incorporation of new information may activate a new line of reasoning, with a stronger conclusion that may revoke the previously held conclusions.

However the advantages of argumentation for representing non-monotonicity are not without a downside, namely, a quite radical departure from orthodox logic. Although this estrangement is natural, given the inability of orthodox logic to handle the incorporation of new information, many interesting characteristics of the orthodox approach that are worth preserving are lost in argumentation systems. For example, argumentation systems lack a semantics in the usual logical sense; instead, they are interpreted by an algebra of notational devices which determines the warranted arguments in a given context. These interpretations can be thought of as supplying a pragmatic foundation,

(c) 2006 Kluwer Academic Publishers. Printed in the Netherlands. 
in the sense that the correct behavior of the system is evaluated in terms of how well they yield the "right" (i.e. intuitive) answer" ${ }^{1}$

At around the same time that argumentation systems were introduced, another line of attack on the problem of representing non-monotonicity began to achieve success in logic. The late Jon Barwise, as a culmination of his work of two decades, introduced (in joint work with Jerry Seligman) a framework to represent the flow of information in distributed systems (Barwise and Seligman, 1997). By a simplification of traditional logic, in order to represent a wide class of contexts in which the information about one component has implications for the information about another, the logic of information flow succeeded in providing an alternative representation of commonsense reasoning.

In an audacious move, Barwise made the claim that reasoning should be put in a framework suitable for the study of physical systems (including the use of numerical intervals for the values of variables). In Barwise and Seligman's framework, non-monotonicity obtains as a result of changes in the background conditions of reasoning processes about spaces of states. This procedure allowed him to keep a logical formalism with the desired properties of commonsense reasoning.

Our goal in this paper is to get the best of both worlds. While, on one hand, argument systems seem more natural to represent some natural features of reasoning, Barwise and Seligman's logic of information flow provides a powerful logical apparatus. We will show that a very abstract system of argumentation, developed by P.M.Dung (Dung, 1995), can be seen as an instance of Barwise's commonsense reasoning in state spaces. On the other hand, this embedding bites back: the different criteria of selection of conclusions detected by Dung may be also incorporated in Barwise's scheme. These criteria represent different epistemic attitudes, like skepticism or credulity. By representing these attitudes as different ways in which background conditions may be structured, we introduce a conceptual innovation to Barwise and Seligman's framework. They conceived only one criterion according to which background conditions may be ordered. In this extensions, commonsense reasoning, according different criteria, may lead to different orderings of background conditions.

In section 2 we present the fragment of Barwise and Seligman's theory that we shall use for the characterization of commonsense reasoning. In section 3 we will discuss briefly Dung's formalization of argument systems. In section 4 we will present our embedding of Dung's system

\footnotetext{
1 There are some paradigmatic problems, with a clear intended answer, that are used to test the performance of argument systems. The best known are about the flying ability of a penguin, once one accepts that penguins are birds and that birds fly.
} 
into Barwise and Seligman's. Moreover, we will show how, in turn, the later framework becomes expanded, allowing us to represent several criteria of reasoning.

\section{Local Logics and Commonsense Reasoning}

Barwise and Seligman developed a theory of information flow in which the notion of local logic plays a fundamental role in the modeling commonsense reasoning. The starting idea is that certain theoretical entities called 'tokens' or 'situations' ${ }^{2}$ are classified by types.

DEFINITION 2.1. A classification is a tuple $\mathcal{C}=\langle\operatorname{Sit}(\mathcal{C}), \operatorname{typ}(\mathcal{C})$, $\left.\models_{\mathcal{C}}\right\rangle$ where $\operatorname{Sit}(\mathcal{C})$ is a non-empty set of objects called the situations of $\mathcal{C}, \operatorname{typ}(\mathcal{C})$ is a set of objects called the types of $\mathcal{C}$, and $\models_{\mathcal{C}}$ is a binary relation on $\operatorname{Sit}(\mathcal{C}) \times \operatorname{typ}(\mathcal{C})$.

Each classification leads to a "theory" and a "local logic". If $\mathcal{C}$ is a classification, a theory $T$ on $\mathcal{C}$ is a set of sequents $\Gamma \vdash_{\mathcal{C}} \Delta$, such that $\Gamma, \Delta \subseteq \operatorname{typ}(\mathcal{C})$, and for every situation $s \in \operatorname{Sit}(\mathcal{C})$, if $s \models \gamma$ for every $\gamma \in \Gamma$ then $s=\delta$ for some $\delta \in \Delta$. These sequents are called the constraints of the theory. A theory is regular iff each constraint verifies the properties of identity, weakening and global cut (see (Barwise and Seligman, 1997): 119). A local $\operatorname{logic} \mathcal{L}$ on $\mathcal{C}$ consists of the classification $\mathcal{C}$, a regular theory $T$ and a subset $N_{\mathcal{L}} \subseteq \operatorname{Sit}(\mathcal{C})$ of situations which satisfy all the constraints in $T$, called the normal situations. A local logic is sound if every situation of $\mathcal{C}$ is normal, and it is complete if $T$ contains all the constraints satisfied by all the normal situations. Theories and local logics derived from a fixed classification can be ordered partially. For regular theories $T_{1}, T_{2}$ over $\mathcal{C}, T_{1} \sqsubseteq T_{2}$ iff every constraint of $T_{1}$ is also a constraint of $T_{2}$. Moreover, for local logics $\mathcal{L}_{1}$ and $\mathcal{L}_{2}$ in a fixed classification $\mathcal{C}, \mathcal{L}_{1} \sqsubseteq \mathcal{L}_{2}$ iff $T_{1} \sqsubseteq T_{2}$ for the corresponding theories $T_{1}$ of $\mathcal{L}_{1}$ and $T_{2}$ of $\mathcal{L}_{2}$, and $N_{\mathcal{L}_{2}} \subseteq N_{\mathcal{L}_{1}}$.

These formal tools can be applied to the study of non-monotonicity in commonsense reasoning. For instance, we can take the state space of a system, say a flashlight, and give a classification in which the situations describe instances of the flashlight and the types are events, i.e., sets of states which have common attributes (for example, the states where the flashlight's batteries are charged, those where the bulb is burned, and so on). A commonsense theory of flashlights could contain constraints such as 'if the switch is on then the bulb is

\footnotetext{
2 In (Barwise and Seligman, 1997) they use the term 'tokens' while Barwise refers to 'situations' in (Barwise, 1999). We use the later expression from now on.
} 
lit' (let us symbolize this with ' $\{$ On (switch $)\} \vdash\{$ Lit(bulb) $\})$ '. Under "normal" conditions we can accept this constraint as valid, while exceptional situations, for example, those in which the batteries are not charged, would be considered "abnormal" and the constraint not valid. Moreover, the theory is not regular for such situations since weakening is violated: $\{$ On (switch), $\neg$ Charged(batteries) $\} \forall\{L i t($ bulb $)\}$. Hence, the local logic for "normal" flashlights is sound only if the batteries are charged. non-monotonicity can be viewed in this model as arising from the transition from one local logic to another: usually we assume the local logic of normal flashlights with charged batteries, but when this "background" condition changes we pass to the local logic of the flashlights with batteries without charge.

A particular kind of local logic can be derived in terms of state space models. The state of a system in a given moment can be described by selecting some parameters and giving the value of those parameters in that moment. Clearly, each situation (particular instance) of a system has exactly one state (even when some states could not correspond to any situation).

DEFINITION 2.2. A state space (classification) $S=\left\langle\operatorname{Sit}(S), \Omega, \models_{S}\right\rangle$ classifies the situations $\operatorname{Sit}(S)$ by means of the types in $\Omega$, called states. Each situation has exactly one state in $\Omega$.

As any other classification, a state space classification can be associated to a local logic:

DEFINITION 2.3. The canonical local logic based on the state space $S, \log (S)$, consists of the following items:

- A classification of events $\operatorname{Evt}(S)=\left\langle\operatorname{Sit}(S), 2^{\Omega}, \models_{E v t(S)}\right\rangle$.

- A regular theory $T h(\log (S))$ in which each constraint is of the form $\Gamma \vdash_{T h(\log (S))} \Delta$, meaning that each situation $s \in \operatorname{Sit}(S)$ that is classified by every event in $\Gamma$ becomes classified by at least one event in $\Delta$.

- The set of situations $\operatorname{Sit}(S)$, which are all assumed to be normal.

Events are useful to represent sets of states sharing some common attributes. Each state $\sigma \in \Omega$ classifies a situation $s \in \operatorname{Sit}(S)$, and this classification can be extended in a straightforward form: $s$ is also classified by any event $\alpha \in 2^{\Omega}$ such that $\sigma \in \alpha$. Since to say that $s$ is classified by an event $\alpha$ is equivalent to indicate that there exists a state $\sigma \in \alpha$ that classifies $s$ in $S$, we have that $\Gamma \vdash_{T h(\log (S))} \Delta$ if and only if $\bigcap_{\alpha \in \Gamma} \alpha \subseteq \bigcup_{\beta \in \Delta} \beta$. Notice that since each situation is normal, $\log (S)$ 
is sound. That is, every situation in $\operatorname{Sit}(S)$ verifies each constraint in $\operatorname{Th}(S)$.

$\log (S)$ is not the only logic that can be defined over the classification $S$ (or its extension $\operatorname{Evt}(S)$ ):

DEFINITION 2.4. A local logic $\mathcal{L}$ over $S$ is called a $S$-logic if it verifies that $\log (S) \sqsubseteq \mathcal{L}$, i.e. for each pair $\Gamma, \Delta \subseteq \operatorname{Evt}(S), \Gamma \vdash_{T h(\log (S))}$ $\Delta$ implies that $\Gamma \vdash_{T h(\mathcal{L})} \Delta$ while the normal situations of $\mathcal{L}$ are $N_{\mathcal{L}} \subseteq$ $\operatorname{Sit}(S)$. A state $\sigma \in \Omega$ is $\mathcal{L}$-consistent if $\{\sigma\} \forall_{T h(\mathcal{L})} \emptyset$, that is, if every state is the state of some situation.

Any $S$-logic $\mathcal{L}$ over a state space $S$ determines a sub-space $S_{\mathcal{L}} \subseteq$ $S$. In fact, the normal situations of $\mathcal{L}$ are the situations that become classified by the states in $S_{\mathcal{L}}$. This fact is more precisely characterized by the following proposition:

PROPOSITION 2.1. If $S$ is a state space and $\mathcal{L}_{1}$ and $\mathcal{L}_{2}$ are two $S$-logics, $\mathcal{L}_{1} \sqsubseteq \mathcal{L}_{2}$ if and only if $S_{\mathcal{L}_{2}} \subseteq S_{\mathcal{L}_{1}}$.

Proof. See (Barwise and Seligman, 1997): 196-197.

This relation becomes particularly interesting for the analysis of nonmonotonicity. Given a general description of a state of affairs in terms of a state space and an intended logic over it, some realizations (the incorporation of new information, for example) may force to change the representation to another logic. Two possible reasons for this may be possible:

- If a new situation $s^{\prime}$ is discovered to be normal.

- If a constraint, previously valid in the theory, is no longer accepted.

In more precise terms, assume that we have a $S$-logic $\mathcal{L}$, with theory $\operatorname{Th}(\mathcal{L})$ and normal situations $N_{\mathcal{L}}$. In the first case, we have that $N_{\mathcal{L}} \subset$ $N_{\mathcal{L}} \cup\left\{s^{\prime}\right\}$. That is, we have a new logic $\mathcal{L}^{\prime}$, such that $\mathcal{L}^{\prime} \sqsubseteq \mathcal{L}$. In the second case we have that, if a constraint $\Gamma \vdash_{T h(\mathcal{L})} \Delta$ is no longer accepted, we have a new logic $\mathcal{L}^{\prime}$ such that $T h(\mathcal{L})=T h\left(\mathcal{L}^{\prime}\right) \cup\left\{\Gamma \vdash_{T h(\mathcal{L})}\right.$ $\Delta$ \}. This means, again, that $\mathcal{L}^{\prime} \sqsubseteq \mathcal{L}$. That is:

PROPOSITION 2.2. Given a state space $S$, the incorporation of a new normal situation $s^{\prime}$ to a theory $\mathcal{L}$ leads to a new theory $\mathcal{L}^{\prime}$ such that there exist $\Gamma, \Delta \subseteq \operatorname{Evt}(S)$ that verify $\Gamma \vdash_{T h(\mathcal{L})} \Delta$ but $\Gamma \forall_{T h\left(\mathcal{L}^{\prime}\right)} \Delta$.

Proof. Trivial. Assume that all the constraints in $\mathcal{L}$ remain valid in $\mathcal{L}^{\prime}$. 
Then, the normal situations for $\mathcal{L}$ keep verifying all the constraints in $\mathcal{L}$. That is $N_{\mathcal{L}}=N_{\mathcal{L}^{\prime}}$. Absurd, since we assume that $N_{\mathcal{L}^{\prime}}=N_{\mathcal{L}} \cup\left\{s^{\prime}\right\}$.

Of course, the dual of this result is also valid. That is, the retraction of a situation deemed normal may lead to the validity of a previously non-accepted constraint.

As said above, if a logic $\mathcal{L}$ changes to a logic $\mathcal{L}^{\prime}$ such that $\mathcal{L}^{\prime} \sqsubseteq \mathcal{L}$, this move is paralleled in a change of sub-spaces, namely from $S_{\mathcal{L}_{1}}$ to $S_{\mathcal{L}_{2}}$ such that $S_{\mathcal{L}_{2}} \subseteq S_{\mathcal{L}_{1}}$. A way of characterizing the sub-spaces is by some conditions (other than the constraints) verified by each state in them. That is, we are looking for some background conditions defining those sub-spaces. More precisely:

DEFINITION 2.5. A background condition $B$, on a state space $S$, is a condition satisfied by some situations, classified by states $\sigma$, such that the set of all these states constitutes a sub-space $S_{B}$.

The proper characterization of what $B$ is, depends on the state space under consideration. Even with this generality it is clear that the following claim is valid:

PROPOSITION 2.3. For each background condition $B$ on $S$ there exists a $\operatorname{logic} \log \left(S_{B}\right)$ such that:

1. The $\log \left(S_{B}\right)$-consistent states are those satisfying $B$.

2. If $\Gamma, \Delta \subseteq \operatorname{Evt}(S), \Gamma \vdash_{T h\left(\log \left(S_{B}\right)\right)} \Delta$ if and only if for every state $\sigma$ satisfying $B, \sigma \in \alpha$ for all $\alpha \in \Gamma$ implies that $\sigma \in \beta$ for some $\beta \in \Delta$.

3. The normal situations are those satisfying $B$.

Proof. The first property of $\log \left(S_{B}\right)$ follows from the definition of background conditions since each $\log \left(S_{B}\right)$-consistent state $\sigma$ is the state of a situation, one in which $B$ is satisfied. The second condition just follows from the fact that the constraints in $\operatorname{Th}\left(\log \left(S_{B}\right)\right)$ are those that define a sub-space $S_{B}$ and therefore can be characterized by a $S$-logic $\mathcal{L}$ in which constraints $\Gamma \vdash_{T h(\mathcal{L})} \Delta$ are defined as $\bigcap_{\alpha \in \Gamma} \alpha \subseteq$ $\bigcup_{\beta \in \Delta} \beta$. Finally, since this $S$-logic characterizes only those states in $S_{B}$, which classify situations that verify $B$, these situations are all normal.

If we can endow the set of background conditions with a partial order $\leq$ such that $B_{1} \leq B_{2}$, meaning that $B_{2}$ puts more stringent conditions than $B_{1}$, i.e., there are less (or equal) number of situations verifying $B_{2}$ than $B_{1}$. More precisely: 
DEFINITION 2.6. If $B_{1} \leq B_{2}$ then $S_{B_{2}} \subseteq S_{B_{1}}$.

LEMMA 2.1. If $B_{1} \leq B_{2}$ then $\log \left(S_{B_{1}}\right) \sqsubseteq \log \left(S_{B_{2}}\right)$.

Proof. Straightforward from the previous proposition and the definition of $\leq . \square$

\section{Defeasible argumentation}

As we said at the beginning of this paper, argument systems provide an alternative representation of reasoning processes in which non-monotonicity is a natural feature. In these systems, non-monotonicity arises as the result of the dynamics of deliberation, in which arguments are compared and may be defeated by other arguments. If new attacking arguments are introduced in a deliberation, the conclusions that were accepted before (because their supporting arguments were not defeated) may be revoked and replaced by other conclusions. So, for example, an argument concluding that the bulb of the flashlight is lit because the switch is on, may be not defeated in some argumentative situation where no opposing arguments defeat it. But if some opposing argument is introduced, for instance one concluding that the batteries are not charged, then the argument supporting that the bulb is lit would become defeated in the new situation.

To get a clear understanding of the aims of the defeasible argumentation approach note that its basic units of analysis are arguments, but not their component propositions. So, a proposition becomes justified or not just because of the status of acceptability of its supporting arguments but not because of its soundness (or any other proof-theoretical feature).

We will follow (Dung, 1995) in his abstract characterization of an argument system.

DEFINITION 3.1. An argument system is a pair $A S=\langle A R, \gg\rangle$, where $A R$ is a set which elements are called 'arguments', and $\gg$ is an arbitrary binary relation among arguments called 'defeat'. $A \gg B$ is interpreted as ' $A$ defeats $B$ '. . (From now on, unless explicitly claimed otherwise, we always refer to an arbitrary but fixed argument system $A S=\langle A R, \gg\rangle$.)

\footnotetext{
3 Dung uses the term 'attack' instead of 'defeat'. We prefer the later, since 'attack' could suggest a symmetric relation. We do not assign a special meaning to 'defeat', but it can be understood as being either objective, or consensually accepted, or decided by a referee, etc.
} 
Dung assumes that a rational agent accepts an argument $A$ if the agent can defend $A$ defeating all the defeaters of $A$. Moreover, a "conflictfree" set of arguments is admissible for a rational agent if all its arguments are acceptable.

DEFINITION 3.2. (Dung, 1995)

1. An argument $A \in A R$ is acceptable in a set $S$ of arguments iff for all $B \in A R$, if $B \gg A$ then $C \gg B$, for some $C \in S$.

2. A set $S$ of arguments is admissible iff $A \ngtr B$ for all $A, B \in S$, and $A$ is acceptable with respect to $S$, for all $A \in S$.

EXAMPLE 3.1. Let $A R=\{A, B, C\}$ with $A \gg B$ and $B \gg C$. Then $\emptyset$ and $\{A, C\}$ are admissible.

Different intuitions about the defensibility of a set of arguments are formalized by Dung, by defining different kinds of "extensions". For Dung, extensions are not supersets of $A R$, as it is natural to think, but the subsets of $A R$ that contain defensible arguments.

DEFINITION 3.3. A set of arguments $S \subseteq A R$ is:

- a stable extension iff $A \ngtr B$ for all $A, B \in S$, and for all $A \notin S$, there exist some $B \in S$ such that $B \gg A$;

- a preferred extension iff $S$ is a maximal (w.r.t. set inclusion) admissible set;

- a complete extension iff given the function $F(S)=\{A: A$ is acceptable in $S\}, F(S)=S$ (i.e., $S$ is a fixed point of $F$ );

- the grounded extension of $A S$ iff $S$ is the least fixed point of $F$.

These extensions characterize different epistemic attitudes: preferred extensions give the most credulous semantics among the extensions, while the grounded extension (which is unique) gives the most skeptical. On the other hand, stable extensions are the only not well defined (i.e., not every argument system has one). The connections between the different extensions is given by the following result:

LEMMA 3.1.

1. Each stable extension is a preferred extension, but not vice versa.

2. Each preferred extension is a complete extension, but not vice versa. 
3. The grounded extension is the least (w.r.t. set inclusion) complete extension.

4. The complete extensions form a complete semi-lattice w.r.t. set inclusion.

Proof. See (Dung, 1995), Lemma 15 (p. 328) for 1 and Theorem 25 (p. 330) for 2, 3 and 4.

\section{Argumentation State Spaces}

We can model an argument system as a state space, defining a classification on that space and a local logic on that classification. The guiding intuition is that an argument system is an ideal representation of all the arguments that can be entered into a discussion on a certain subject, assuming a fixed defeat relation among arguments. For example, an argument system representing the previous discussion about the flashlight's bulb would contain a symbol for every relevant argument supporting that the bulb is lit or supporting that it is not. The state space of the system contains all the possible situations involving those arguments, where each situation represents a possible particular debate, a possible instance of the discussion.

DEFINITION 4.1. The argumentation state space of $A S=\langle A R, \gg\rangle$ is $S_{A S}=\left\langle\operatorname{Sit}\left(S_{A S}\right), \Omega_{A S}\right\rangle$, where $\operatorname{Sit}\left(S_{A S}\right)=\Omega_{A S}=2^{A R}$ (recall that each situation has exactly one state in $\Omega$; situations and states can be identified each another in this state space).

EXAMPLE 4.1. Assume $A R=\{A, B\}$. The situations of the argumentation state space are $\operatorname{Sit}\left(S_{A S}\right)=\{\emptyset,\{A\},\{B\},\{A, B\}\}$.

PROPOSITION 4.1. The canonical local logic based on the argumentation state space $S_{A S}, \log \left(S_{A S}\right)$, consists of the following items:

- A classification of events $\operatorname{Evt}\left(S_{A S}\right)=\left\langle\operatorname{Sit}\left(S_{A S}\right), 2^{\Omega} A S\right\rangle$.

- A regular theory $T h\left(\log \left(S_{A S}\right)\right)$ in which each constraint is of the form $\Gamma \vdash_{T h\left(\log \left(S_{A S}\right)\right)} \Delta$, meaning that each situation $s \in \operatorname{Sit}\left(S_{A S}\right)$ that is classified by every event in $\Gamma$ becomes classified by at least one event in $\Delta$.

- The set of situations $\operatorname{Sit}\left(S_{A S}\right)$, which are all normal.

Proof. Immediate from definition 2.3. 
We are looking for a more interesting local logic than just the canonical. In a given debate, it is important to know on which arguments we should count on to defend some critical argument. So we are interested in the local logic of all the states satisfying a specific background condition, to wit, all the states in which that critical argument can be defended. Here is the crucial idea for rationalizing non-monotonicity, which can be illustrated with the following example. Suppose we want to defend an argument $A$. This argument would be defended unless another argument defeating it were defended. If $A$ is defeated in the same argumentation state by an argument $B$, then there must in time be another argument $C$ defeating $B$ in that state. Otherwise, $A$ could not be defended in that argumentation state. Assume $A, B$ and $C$ are the only three arguments in the system. It is clear that in the states $\sigma$ in which $A$ is defensible it is true that if $B \in \sigma$ then also $C \in \sigma$. Regularities like this one give us a local logic for the sub-space of all the states in which $A$ is defensible. As this sub-space is determined by the fact that $A$ is defensible in all its states, we will say that the defensibility of $A$ is a background condition satisfied by all those states.

In order to give a formal definition of 'background conditions', we have to fix the meaning of 'defensibility'. As discussed in the preceding subsection, one can think about different criteria. The extensions defined by Dung, stable, preferred, grounded and complete, give us four possible meanings of defensibility and hence four possible kinds of background conditions.

DEFINITION 4.2. Given an argument system $\langle A R, \gg\rangle, B \subseteq A R$ is a background condition satisfied by an argumentation state $\sigma \in \Omega_{\langle A R, \gg\rangle}$ iff $B \subseteq D \subseteq \sigma$ for some set $D$ of arguments that is defensible in $\left\langle\sigma, \gg_{{ }_{\sigma}}\right\rangle$. As defensibility is defined in terms of extensions, $B$ can be super-indexed according to the kind of extension considered, as follows:

$-B^{s}$, if $D$ is stable;

$-B^{p}$, if $D$ is preferred;

$-B^{g}$, if $D$ is grounded;

$-B^{c}$, if $D$ is complete.

By proposition 2.3 we know that every background condition $B$ will determine a sub-space of the argumentation state space, to wit, the sub-space $S_{B}$ of all the states that satisfy the background condition $B$. In turn, $S_{B}$ will determine the local $\operatorname{logic} \log \left(S_{B}\right)$ of all the constraints satisfied by all the states in $S_{B}$. Since background conditions, as defined for argumentation state spaces, can be partially ordered by set 
inclusion, then we have that if $B_{1} \subseteq B_{2}$ then $\log \left(S_{B_{1}}\right) \sqsubseteq \log \left(S_{B_{2}}\right)$, as Lemma 2.1 ensures. That is, as background conditions become more stringent (i.e., more arguments need to be defended), so the associated logics increase their strength (i.e., more constraints are added and there are less states that are normal).

EXAMPLE 4.2. Consider the system $A S=\langle\{A, B, C\},\{C \gg B$, $B \gg A\}\rangle$ where we interpret $A$ : 'the flashlight's bulb would be lit if you turn the switch on', $B$ : 'the flashlight's bulb would not be lit if I turn the switch on, because the batteries are not charged', and $C$ : 'the flashlight's bulb would be lit if you turn the switch on, because I charged the batteries yesterday'. Assume our intuition is that arguments are defensible if they belong to a preferred extension. Then the states verifying the background condition $\{A\}^{p}$ ("how can I defend that the bulb would be lit?") are $\left\langle\{A\}, \gg_{\mid\{A\}}\right\rangle,\left\langle\{A, C\}, \gg_{\{A, C\}}\right\rangle$ and $\langle\{A, B, C\}$, $\gg$ since all of them have a preferred extension including $\{A\}$. Now, for any argument $A$ let us define the event $[A] \in 2^{\Omega_{A S}}$ as the set of all the states containing the argument $A$, that is, $[A]=\{\sigma: A \in \sigma\}$. Then $[B] \vdash_{\{A\}^{p}}[C]$ is a constraint in $\log \left(S_{\{A\}^{p}}\right)$, the local logic determined by the sub-space $S_{\{A\}^{p}}$. That constraint says that if $B$ (which defeats $A$ ) belongs to the states where $A$ is defensible, then $C$ (which defeats $B$ ) also belongs to those states. We can interpret this as indicating that to defend the claim that the bulb would be lit if the switch is on, one must be able to defend the claim that the batteries are charged, otherwise it could not be defended at all.

We can also induce an ordering of the local logics determined by background conditions according to the different intuitions behind defensibility (i.e., the different kinds of extensions considered). This will give us a complete picture of the connections among the different local logics obtained by the respective intuitions.

\section{DEFINITION 4.3.}

$-B_{1} \leq_{s} B_{2}$ iff $B_{1}^{s} \subseteq B_{2}^{s}$

$-B_{1} \leq_{p} B_{2}$ iff $B_{1}^{p} \subseteq B_{2}^{p}$;

$-B_{1} \leq_{g} B_{2}$ iff $B_{1}^{g} \subseteq B_{2}^{g}$;

$-B_{1} \leq_{c} B_{2}$ iff $B_{1}^{c} \subseteq B_{2}^{c}$.

Similarly,

$-\log \left(S_{B_{1}}\right) \sqsubseteq_{s} \log \left(S_{B_{2}}\right)$ if $B_{1} \leq_{s} B_{2} ;$ 
$-\log \left(S_{B_{1}}\right) \sqsubseteq_{p} \log \left(S_{B_{2}}\right)$ if $B_{1} \leq{ }_{p} B_{2}$;

$-\log \left(S_{B_{1}}\right) \sqsubseteq g \log \left(S_{B_{2}}\right)$ if $B_{1} \leq_{g} B_{2}$;

$-\log \left(S_{B_{1}}\right) \sqsubseteq_{c} \log \left(S_{B_{2}}\right)$ if $B_{1} \leq_{c} B_{2}$.

\section{THEOREM 4.1.}

1. If $B_{1} \leq_{s} B_{2}$ then $B_{1} \leq_{p} B_{2}$.

2. If $B_{1} \leq_{p} B_{2}$ then $B_{1} \leq_{c} B_{2}$.

3. If $B_{1} \leq_{g} B_{2}$ then $B_{1} \leq_{c} B_{2}$.

Proof. Let us prove 1, since the proofs for the remaining cases are similar. Assume $B_{1} \leq_{s} B_{2}$. By definition, $B_{1}^{s} \subseteq B_{2}^{s}$. Then, there exists a stable extension $S$ such that $B_{1} \subseteq B_{2} \subseteq S$. By Lemma 3.1, $S$ is preferred and hence $B_{1}^{p} \subseteq B_{2}^{p}$, that by definition implies $B_{1} \leq{ }_{p} B_{2}$.

COROLLARY 4.1.

1. If $\log \left(S_{B_{1}}\right) \sqsubseteq_{s} \log \left(S_{B_{2}}\right)$ then $\log \left(S_{B_{1}}\right) \sqsubseteq_{p} \log \left(S_{B_{2}}\right)$.

2. If $\log \left(S_{B_{1}}\right) \sqsubseteq_{p} \log \left(S_{B_{2}}\right)$ then $\log \left(S_{B_{1}}\right) \sqsubseteq_{c} \log \left(S_{B_{2}}\right)$.

3. If $\log \left(S_{B_{1}}\right) \sqsubseteq_{g} \log \left(S_{B_{2}}\right)$ then $\log \left(S_{B_{1}}\right) \sqsubseteq_{c} \log \left(S_{B_{2}}\right)$.

Proof. Immediate from Theorem 4.1 and Lemma 2.1.

\section{Conclusions}

We presented in this paper an embedding of argumentation systems in the framework of local logics based on state spaces. We conceive argumentation systems as supporting different states in a deliberation or discussion. The relations among these states is captured by means of local logics.

We saw that the embedding is quite natural, providing a clear semantics for argumentation while at the same time keeping its properties. In particular, the non-monotonicity that arises in argument systems obtains in the corresponding local logics as the transition from a background condition to another.

Background conditions, which Barwise and Seligman conceived as partially ordered according to a single criterion, may be ordered, in our case, in four different forms. Each partial order corresponds to a different epistemic attitude (a criterion of defensibility of arguments). 
These partial orders, in turn, have a close relation among them, as a reflection of the properties of the extensions of argument systems that obey to the criteria of defensibility.

The possibility of having different orderings of the background conditions expands the reach of Barwise and Seligman's framework, since it allows us to represent the fact that commonsense reasoning may conform to different epistemic attitudes. In fact, while here we consider only attitudes in term of skepticism, many other alternatives are readily available. Therefore, our framework may provide a general taxonomy of reasoning, which is matter for further work.

\section{Acknowledgments}

The first author is in debt to Professor Dr. Shahid Rahman for enriching debates during his visit to Bahía Blanca, Argentina. Both authors thank an anonymous referee for a deep philosophical analysis of our aim. This paper was partially supported by Secretaría de Ciencia y Técnica (Secretary of Science and Technics) of the Universidad Nacional del Sur.

\section{References}

Barwise, J., 1999, "State spaces, local logics and non-monotonicity," in Logic, Language and Computation, vol. 2, L.Moss, J.Ginzburg and M.de Rijke, eds., Stanford: CSLI Publications.

Barwise, J. and Seligman, J., 1997, Information Flow: The Logic of Distributed Systems, Cambridge, MA: Cambridge University Press.

Dung, P. M., 1995, "On the acceptability of arguments and its fundamental role in non-monotonic reasoning, logic programming, and $n$-person games," Artificial Intelligence 77, 321-357.

McCarthy, J., 1980, "Circumscription-A form of non-monotonic reasoning," Artificial Intelligence 13, 27-39.

McCarthy, J. and Hayes, P., 1969, "Some philosophical problems from the standpoint of Artificial Intelligence," in Machine Intelligence 4, B. Meltzer and D. Mitchie, eds., Edimburg University Press.

McDermmot, J. and Doyle, J., 1980, "Non-monotonic Logic I," Artificial Intelligence 13, 41-72.

Lin, F. and Shoham, Y., 1989, "Argument systems: a uniform basis for nonmonotonic reasoning," pp: 245-255 in Proc. of the 1st International Conference on Knowledge Representation and Reasoning, San Mateo, CA: Morgan Kaufmann Publishers.

Loui, R., 1987, "Defeat among arguments: a system of defeasible inference," Computational Intelligence 3 (3), 100-106.

Loui, R., 1998, "Process and policy: resource-bounded non-demonstrative reasoning", Computational Intelligence 14, 1-38. 
Pollock, J., 1987, "Defeasible Reasoning," : Cognitive Science 11, 481-518.

Poole, D., 1988, "A logical framework for default reasoning," Artificial Intelligence 36 (1), 27-47.

Reiter, R., 1980, "A logic for default reasoning," Artificial Intelligence 13, 81-132.

Simari, G. and R. Loui, 1992 "A mathematical treatment of defeasible reasoning and its implementation," Artificial Intelligence 53, 125-157. 
JoLLI.tex; $12 / 05 / 2006 ; 13: 39 ;$ p.16 